\title{
NEUERE DEUTUNGEN VON MITTELEUROPAISCHEN OBERFLÄCHENFORMEN
}

\author{
Von Eduard K. Gerber
}

\begin{abstract}
Von jeher haben die Oberflächenformen der Erde, die uns umgeben, zu Deutungsversuchen angeregt. So wurden früher die Alpen als das Werk ungeheurer personifizierter Schöpfer- und Zerstörungskräfte aufgefaßt. Der moderne Mensch kann sich damit nur dann noch befreunden, wenn es sich um Dichtung handelt; etwa in der Form einer Stelle im "Olympischen Frühling" von CarL Spitteler, die P. NigGri das "Hohe Lied des Petrologen und Geologen» genannt hat ${ }^{1}$. Aber auch der moderne Mensch versucht zu deuten, indem er die Formen als Ausdruck natürlich ablaufender, rational erfaßbarerVorgänge betrachtet. Wir müssen allerdings bekennen, daß einzelne dieser Deutungen, vor allem die ersten Versuche und ganz besonders die allzu vereinfachten populären Ansichten, ebenso phantastisch sind wie die vorrationalistischen, ohne deren künstlerischen Wert zu besitzen, ja, daß bis jetzt überhaupt kein einziger Deutungsversuch von Großformen, auch der ernsthafteste und beste, in jeder Beziehung einem streng wissenschaftlichen Maßstab standhalten kann, und daß alle Anstrengungen in dieser Richtung nur sehr gewagte Extrapolationen sind. Und trotzdem haben sie ihre Berechtigung, weil dadurch und gerade dort, wo die Deutungen versagen, der Grundlagenforschung neue Impulse und neue Fingerzeige für die Forschungsrichtung gegeben werden. Diese Basis, von der aus neue Deutungen versucht werden können, zu erweitern, muß immer wieder eine dringliche Aufgabe sein. Sie kann auf zwei Arten erweitert werden, erstens durch immer genaueres Erfassen heute beobachtbarer Vorgänge, dann durch Verfeinerung der Theorie dieser Vorgänge. Im Vordergrund der Formdeutung der Alpen standen und stehen noch immer die fluviatilen und glazialen Vorgänge. Das wird schlagwortartig zusammengefaßt durch den Titel: Fluß- und Eiswerk in den Alpen ${ }^{2}$. Fluviatile Erosion ist die mehr linear, glaziale Erosion die mehr flächenhaft wirkende Erosionsart. Nun wissen wir, daß die Oberfläche sich auch verändern kann, ohne daß diese zwei Erosionsarten beteiligt sind, nämlich durch tektonische Vorgänge, auf die aber hier nicht eingegangen werden soll, und durch Verwitterung. Diese ist allerdings an vielen Stellen gering; sehen wir doch gegenwärtig noch völlig intakte Gletscherschliffe oder vom Fluß geschaffene Strudellöcher an Felsflächen, die seit langem vom Gletscher oder Wasser verlassen sind. Und doch kennen wir in den Alpen gewaltige Schutthalden, die durch Verwitterung entstanden sind. 1874 hat Albert Heim auf die Schwarzweißlinie (Schnee-Fels) hingewiesen, wo die Verwitterung sehr rasch vor sich geht. Ja, er zeichnet an dieser Stelle in einem "allgemeinen Querprofil" sogar eine Verflachungszone (Terrasse), die also durch Verwitterung entstanden ist ${ }^{3} .1910$ hat in einem Entwicklungsschema
\end{abstract} F. NussBaum ungefähr gleichhoch liegende Verflachungen als Karterrassen gedeutet ${ }^{4}$.

Schutthaldenbildungen unter Felswänden wurden nun, wie wir schon einmal in dieser Zeitschrift berichteten, in neuerer Zeit vor allem von O. LeHMANN und J. P. BAKKER studiert. Sie zeigten, daß beim Zurückwittern einer Felswand unter dem -Schuttmantel eine stark bauchig vorgewölbte Felswand entsteht (grob ausgedrückt in Form einer Parabel; genau ist es eine logarithmische Kurve) ${ }^{5}$. Daß solche Formen unter einer Schutthalde entstehen, ist theoretisch absolut einwandfrei nachgewiesen. Doch sehen wir natürlich praktisch diese Formen gar nicht, da sie ja vom Schutt verhüllt sind. Sie werden erst sichtbar, wenn zur Denudation Erosion dazutritt, glaziale oder fluviatile. LEHMANN, der sich in diese Probleme am besten eingearbeitet hatte, hat leider nur einen Torso hinterlassen, in dem zwar eine Fülle von Anregungen steckt ${ }^{\mathrm{B}}$.

Jüngst hat nun aber BAKKER unter anderm eine für die Alpen interessante Deutung gewagt. Er stellt dem errechneten, bauchig vorgewölbten Felsprofil ein Bild des Grimselgebietes gegenüber. Auf diesem sehen wir über einem sehr steil aus der Talsohle aufsteigenden Gehänge eine stark geneigte Verflachung,

1 P. NiggLr: Schulung und Naturerkenntnis, Zürich 1945. S. 188.

2 J. SöLCH: Fluß- und Eiswerk in den Alpen zwischen Otztal und St. Gotthard. Petermanns Mitteilungen, Ergänzungsheft 219/220. Gotha 1935.

3 Albert Heim: Einiges über Verwitterungsformen der Berge. Neujahrsblatt der Naturforschenden Gesellschaft Zürich auf das Jahr 1874, Figur 5.

4 F. Nussbaum: Die Täler der Schweizer Alpen. Bern 1910.

5 O. Lehmann: Morphologische Theorie der Verwitterung von Steinschlagwänden. Vierteljahresschrift der Naturforschenden Gesellschaft Zürich 1933, S. 83-126. - J. P. BAKKER und J. W. N. LE Heux: Projektive-geometric treatment of Lehmann's theory of the transformation of steep mountain slopes. Proc. Kon. Ned. Acad. van Wetenschappen. Amsterdam 1946, S. 533 - 547. - Dieselben: Theory on central rectilinear recession of slopes. Proc. Kon. Ned. Acad. van Wetenschappen 1947, S. $959-966$ und S. $1154-1162$.

B O. LehmanN: Die morphologische Wirksamkeit und topographische Verborgenheit von Verwerfungen. Posthum. Vierteljahresschrift der Naturforschenden Gesellschaft Zürich, LXXXVI 1941, S. $256-291$. 
die Trogschulter, die nach oben in die steilen, zackig verwitterten Gratformen übergeht? ${ }^{\text {? }}$ Die Ähnlichkeit dieses Hangprofils mit dem LeHMANN-BAKKerschen Denudationsprofil ist auffallend. Ähnliche Bilder aus hochalpinen Tälern ließen sich noch eine ganze Reihe geben. Ist diese Ähnlichkeit nun rein zufällig oder entsprechen gewisse Trogschultern den theoretisch gut fundierten Denudationsprofilen? BAKKER wagt diese Extrapolation. Sie bedingt aber einige Annahmen, denen nicht einfach bedenkenlos zuzustimmen ist, die aber der Überprüfung wohl wert sind, nämlich, daß in der Präglazialzeit schon tiefe Täler mit ursprünglich hohen, aber schon weitgehend zurückgewitterten Felswänden bestanden, wobei unter dem angehäuften Schutt trogschulterartige Formen entstanden. In den Eiszeiten mußten dann diese Schuttmassen ausgeräumt und das Gehänge überschliffen werden. Der Annahme solcher alter und außerordentlich tiefer Kerbtäler in einer frühern Zeit (die Datierung müßte noch versucht werden) "widerspricht ja aber alles, was wir sonst wissen " ${ }^{8}$. Gegenüber den frühern Deutungsversuchen, auch den zwei oben erwähnten von Herm und Nussbaum, liegt die Schwäche darin, daß die auffallend gleiche Höhenlage dieser höchsten Verflachungen des Gehänges durch einen solch reinen Denudationsvorgang nicht ohne weiteres erklärt ist.

Immerhin ist der Gedanke gewaltiger Schuttmassen im präglazialen Alpeninnern nicht so neuartig; denn schon 1905 schrieb HeIM: "Die Hauptwirkung der Eiszeit im schweizerischen Alpengebirge besteht darin, daß sie den alt angehäuften Gebirgsschutt hinaustransportiert hat." Und von den neuseeländischen Alpen schreibt er: "... Berge der äußern Ketten sind unter einen Mantel ihres eigenen Schuttes getaucht und an ihrer Sohle hoch in Talaufschüttungen bis über die Knie oder gar bis an die Hüfte eingebettet. Manche Schutthalden reichen vom Fuß der Berge bis an die Gipfel hinauf ${ }^{9}$.»

Starke Verwitterung ist aber nicht nur an die Schwarzweißgrenze gebunden, sondern nach den Untersuchungen von J. BüDEL ein Merkmal des ganzen Periglazialgebietes. Wir müssen annehmen, daß in der Glazialzeit sehr ausgedehnte Flächen der Frostverwitterung und der Solifluktion ausgesetzt waren. Die Schuttwanderung findet unter diesen Bedingungen noch bei Oberflächenneigungen von nur 2-3 Grad statt. Die Untersuchungen BüDELS stïtzen sich nun zwar nicht auf das Alpeninnere und würden eher Schlüsse für unsern Jura und das Mittelland zulassen; aber es ist doch denkbar, daß auch in den Alpen einmal solche Verwitterung stattfand ${ }^{10}$. Das muß zu einer starken Erweiterung des Geltungsbereiches der theoretischen Überlegungen von LehmanN-BakKER führen. Denn sobald Schutt abfließen kann, ohne daß von weiter oben gleichviel oder noch mehr zufließt, muß die Verwitterung tiefer greifen; dort aber, wo mehr zufließt als abfließen kann, muß der Schutt wie ein schützender Mantel die weitere Verwitterung aufheben. $\mathrm{Da} \beta$ damit der Bildung eigenartiger Oberflächenformen, ganz allgemein konvexen Gehängeformen Vorschub geleistet wird, liegt auf der Hand. Wie C. Troll dargelegt hat, ist Solifluktion eine weltweit beobachtbare Erscheinung, und sicherlich würde sich in der Schweiz manche Form damit besser als bis jetzt erfassen lassen ${ }^{11}$.

Auf Grund der Verbreitung von Löß und Dünen, Lehmkeilen, Würge- und Taschenböden usw. in West- und Mitteleuropa hat H. Poser ${ }^{12}$ Klimagebiete der Würmeiszeit abgegrenzt und zu deren Erklärung auch eine Karte der Luftdruckverteilung und Windrichtungen gezeichnet (mit stabilen Hoch über den Eismassen der Alpen und von Skandinavien) und damit den klimatologischen Problemkreis der Periglazialerscheinungen (mit manchen ungelösten Fragen) übersichtlich dargestellt. Auffallenderweise fehlen die schweizerischen Löß- und Dünenvorkommnisse, wohl weil sie teilweise innerhalb der Würmmoränen liegen.

Die hier lediglich in großen Zügen entwickelten Gedanken können den ganzen Problemkreis natürlich nur skizzieren. Gesichert sind die einwandfrei abgeleiteten Formen des Felskernes unter Schutthalden, gesichert sind viele Beobachtungen über Solifluktion. Auch wenn die Trogschultern in keinem Fall als LenmanN-Bakkersche Denudationsprofile gedeutet werden könnten, muß doch bei jedem konvex gerundeten Felsprofil an diese Deutungsmöglichkeit gedacht werden. Ganz allgemein aber ist den Denudationsformen neben den glazialen und fluviatilen Erosionsformen erhöhte Beachtung zu schenken; denn durch Denudation können unter anderm hoch über der Talsohle und ohne primäre Mitwirkung von Fluß oder Gletscher Verflachungen entstehen, die nicht durch Wechsel in der Erosions-

7 J. P. BAKKer: Over tectogene en morfogene Gelijktijdigheid bij de jongere Gebergtevorming in West- en Midden-Europa in het Kader van denudatieve Altiplanatie. Résumé français: Sur la simultanéité morphogénique et tectonique des orogéneses récentes dans l'ouest et le centre de l'Europe considérées dans le cadre de l'altiplanation dénudative. Naturwetenschappelijk Tijdschrift, 30, 1948, S. 3-53.

${ }^{8}$ Briefliche Mitteilung von C. Rathjens, München.

- Albert Heim: Das Säntisgebirge. I. Textband. Beiträge Geologische Karte der Schweiz. Bern 1905.

1n J. BüDEL: Eiszeitliche und rezente Verwitterung und Abtragung im ehemals nicht vereisten Teil Mitteleuropas. Petermanns Mitteilungen, Ergänzungsheft 229. Gotha 1937.

11 C. Troll: Die Formen der Solifluktion und die periglaziale Bodenabtragung. "Erdkunde», I, 1947, S. 162 - 175. - Derselbe: Der subnivale oder periglaziale Zyklus der Denudation "Erdkunde», II, 1948, S. 1-21.

12 H. POSER: Äolische Ablagerungen und Klima des Spätglazials in Mittel- und Westeuropa. "Die Naturwissenschaften", 35, 1948, S. 269-276 und 307-312. - Derselbe: Boden- und Klimaverhältnisse in Mittel- und Westeuropa während der Würmeiszeit. "Erdkunde», II, 1948, S. 53-68. 
geschwindigkeit zu erklären sind, sondern eher mit Akkumulation in der Talsohle oder dem Gehängefuß zusammenhängen.

Wir kommen zum Anfang zurück. In jeder Form liegt der Anreiz zur Deutung. Wenn wir auf eher vernachlässigte Vorgänge aufmerksam machten, so wissen wir, daß sich mit deren weiterm Studium und verbesserten Kenntnissen die Deutungsversuche nicht einfacher gestalten, im Gegenteil eher noch verwickelter werden. Um so phantastischer wirkt die Vorstellung, daß wir mit unsern recht dürftigen Kenntnissen schon endgültige Deutungen geben können. Es ist vielmehr anzunehmen, daß noch Vorgänge eine Rolle spielten, die wir uns gar nicht vorstellen können, weil wir sie gegenwärtig nicht beobachten können, zum Beispiel weil die klimatischen Voraussetzungen dazu fehlen. Diese rein negativen Feststellungen werden aber den Geographen dann nicht entmutigen, wenn für ihn der Mensch auf der gegenwärtigen Erdoberfläche im Vordergrund steht. Er wird dann mit um so größerem Eifer an der andern unvollendeten großen Aufgabe den Bodenformen gegenüber, der Systematik der Formen und ihrer Verteilung auf der Erdoberfläche, arbeiten.

\section{DIE SAMMLUNG FƯR VƠLKERKUNDE DER UNIVERSITAT ZÚRICH IM JAHRE 1948/49}

\section{Mit 2 Textabbildungen}

Im Berichtsjahre bestand die Tätigkeit der Völkerkundesammlung im wesentlichen in der Weiterführung der inneren Ausgestaltung der Schauräume, soweit es die äußerst beschränkten Platzverhältnisse zuließen, ferner in der Determinierung und Katalogisierung von Museumsobjekten, in der Erledigung und Beantwortung von Anfragen seitens verschiedener Schweizer Museen und einzelner Privatpersonen sowie in Führungen von Schulen, Vereinen und anderweitigen Interessengruppen, u. a. von der CIBA, Basel. Eine wissenschaftliche Bearbeitung erfuhren die afrikanischen Schmuckgegenstände, die übrigens

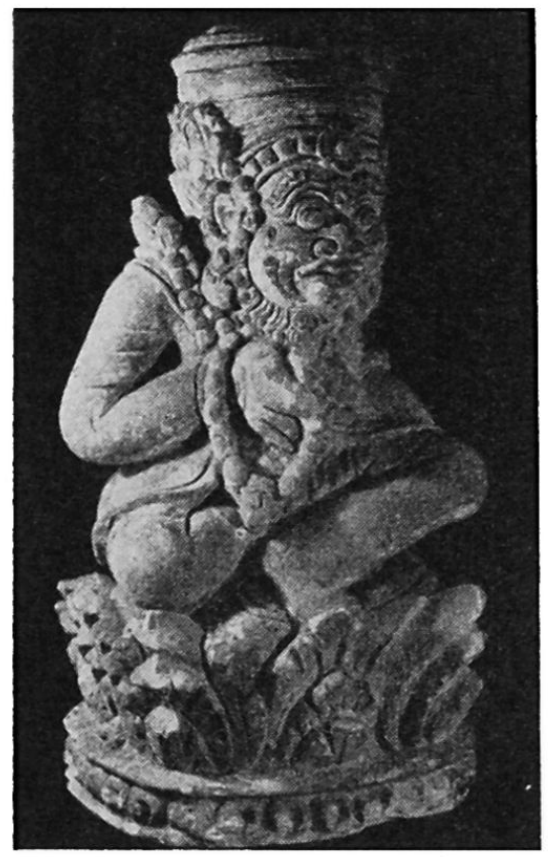

Abb. 1. Steinerne Tempelwächterfigur aus Bali
PHOTOS: E. LEUZINGER, ZURICH

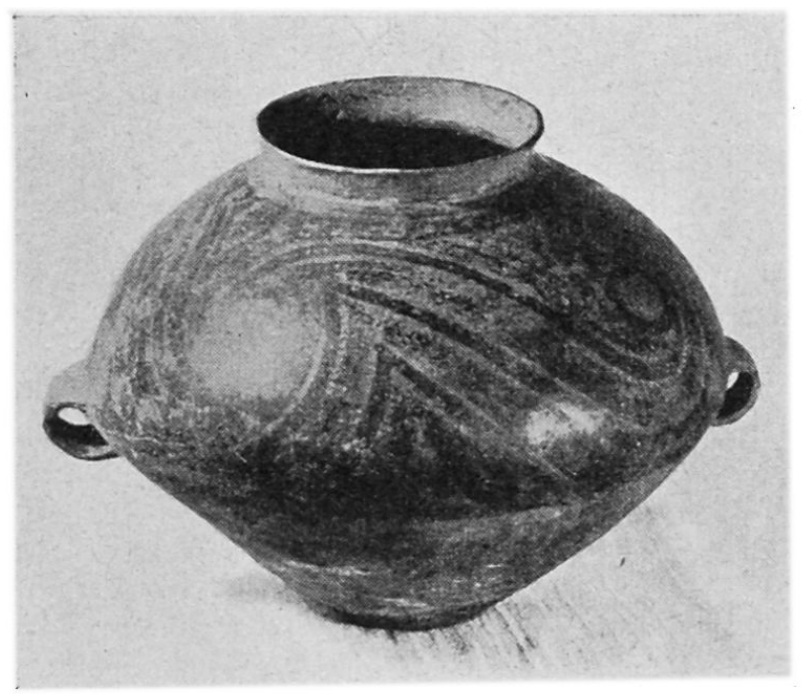

Abb. 2. Kansu-Grabkeramik (Chines. Neolithikum)

auch in einer Pultvitrine neu aufgestellt wurden, sowie die primitiven Batikarbeiten chinesischer Miaotze-Stämme; zwei diesbezügliche Publikationen stehen vor ihrem Abschluß. - Daneben wurde der Sachkatalog der wissenschaftlichen Handbibliothek und der Ausleihedienst von Büchern und Diapositiven an Dozenten und Studenten von der Konservatorin wie bisher weitergeführt.

An der Ausstellung indischer Miniaturen im Basler Kunstgewerbemuseum beteiligte sich die Sammlung mit 13, an derjenigen im Graphischen Kabinett der ETH in Zürich mit 3, an der japanischen Ausstellung des H. Boller in Winterthur mit 2 Objekten; dem Zürcher Pfauentheater wurden außerdem 10 Museumsobjekte leihweise zur Verfügung gestellt. Die Besucherzahl betrug insgesamt 5136 Personen, darunter 43 Schulen und 2 Vereine.

Als Geschenke wurden der Sammlung im Berichtsjahre insgesamt 37 Objekte überwiesen. Donatoren waren die Herren Dr. Billeter, Prof. L. Chazai, Dr. C. Friedländer, Dr. Th. Goldschmidt, 